Estudios deDerecho

Fundada en 1912
UNIVERSIDAD DE ANTIOQUIA 


\title{
Crisis en los sistemas penitenciarios: derechos humanos, hacinamiento y desafíos de las políticas criminales. Una aproximación desde la producción bibliográfica*
}

\section{William Chará Ordóñez ${ }^{* *}$}

\section{Resumen}

Este artículo de revisión tiene como objetivo indagar las investigaciones académicas realizadas sobre los debates alrededor de las crisis carcelarias que develan el hacinamiento y las apuestas por las reformas a las políticas criminales para atender estas situaciones. Para tal fin, se acude a una metodología cualitativa de corte documental a partir de la revisión de la base de datos Scopus. De los 393 resultados obtenidos de esa base de datos, bajo criterios de exclusión como área disciplinar, periodo de estudio y relevancia temática, finalmente se realiza el análisis de 78 documentos. El análisis se realiza en VOSviewer y permite agruparlos en tres categorías: crisis del sistema carcelario, situaciones conflictivas en las cárceles y opciones de respuesta de la política criminal. Los resultados y conclusiones se obtienen luego de un análisis de contenido que permiten advertir que existen tres debates alrededor del tema: los estudios que indican los retos que presentan los sistemas penitenciarios para la reducción del hacinamiento, aquellos que dan cuenta de las situaciones endógenas que evidencian las crisis, y finalmente, las propuestas para las reformas de las políticas criminales. Palabras clave: política criminal; crisis carcelaria; hacinamiento carcelario; derechos humanos.

\section{Crisis in prison systems: human rights, overcrowding, and challenges of criminal policies. An approach from the bibliography}

\begin{abstract}
This review article investigates academic research about the debates surrounding prison crises that expose overcrowding and the bets on criminal policy reforms to address these situations. For this purpose, a documentary analysis as a qualitative research method is used based on the Scopus database review. Of the 393 results obtained, the following exclusion criteria were executed: disciplinary area, study period, and thematic relevance, which let us with 78 viable documents. The analysis was performed in VOSviewer, allowing us to group the papers into three categories: the crisis in the prison system, conflictive situations in prisons, and response options for criminal policy. The results and conclusions are obtained after the implementation of a content analysis, which allowed us to notice that there are three debates around the subject: Studies that indicate the challenges that prison systems present in reducing overcrowding, those that account for the endogenous situations that are evident by the crises, and finally the proposals for criminal policy reforms. Keywords: criminal policy; prison crisis; prison overcrowding; human rights.
\end{abstract}

\section{Crise nos sistemas penitenciários: direitos humanos, superlotação e desafios das políticas criminais. Uma aproximação desde a produção bibliográfica}

\section{Resumo}

Esse artigo de revisão tem como objetivo questionar as pesquisas acadêmicas realizadas sobre os debates relacionados com as crises carcerárias que revelam a superlotação e as tentativas de reformas às políticas criminais para atender estas situações. Para tal propósito, apega-se a um estudo qualitativo de coorte documental partindo da revisão da base de dados Scopus. Dos 393 resultados obtidos dessa base de dados, sob critérios de exclusão como área disciplinar, período do estudo e relevância temática, finalmente foi realizada a análise de 78 documentos. A análise foi feita em VOSviewer e permite organizá-los em três categorias: crise do sistema carcerário, situações de conflito nas presídios e opções de resposta da política criminal. Os resultados e as conclusões foram obtidos depois de uma análise de conteúdo, que permite constatar que existem três debates relacionados ao tema: os estudos que indicam os desafios apresentados pelos sistemas penitenciários para a redução da superlotação, aqueles que se referem às situações endógenas que evidenciam a crise, e finalmente, as propostas para as reformas das políticas criminais.

Palavras-chave: política criminal; crise carcerária; superlotação carcerária; direitos humanos.

* $\quad$ Artículo de revisión. Producto del proyecto: "Estado y políticas públicas en tiempos de crisis en Colombia" adscrito al Grupo de Investigación en Ciencias Sociales y Humanidades GISCH de la Corporación Universitaria Autónoma del Cauca financiado en Convocatoria Interna Uniautónoma 2019.

** Docente titular de la Facultad de Derecho, Ciencias Sociales y Políticas de la Corporación Universitaria Autónoma del Cauca, Colombia. Politólogo (Universidad del Cauca) y Magíster en Sociología (Facultad Latinoamericana de Ciencias Sociales - FLACSO Ecuador). Integrante del Grupo de Investigación en Ciencias Sociales y Humanidades -GISCH-. Miembro de la línea de investigación en estudios de Gobierno y Estado. Correo electrónico: william.chara.o@ uniautonoma.edu.co ORCID: 0000-0002-7558-2047

Cómo citar este artículo: Chará Ordóñez, W. (2021). Crisis en los sistemas penitenciarios: derechos humanos, hacinamiento y desafíos de las políticas criminales. Una aproximación desde la producción bibliográfica. Estudios

de Derecho, 78 (171), 117-138

Doi: 10.17533/udea.esde.v78n171a05

Fecha de recepción: 21/07/2020 Fecha de aceptación: 22/09/2020 


\section{Crisis en los sistemas penitenciarios: derechos humanos, hacinamiento y desafíos de las políticas criminales. Una aproximación desde la producción bibliográfica}

\section{Introducción}

El 21 de marzo "varios internos de la cárcel La Modelo -Bogotá- protestaron por las condiciones inhumanas y la falta de medidas sanitarias frente a la pandemia del COVID-19" (El Tiempo, 2020). Esto desencadenó una respuesta institucional con un saldo de 23 reclusos fallecidos y 83 heridos. Este hecho, en el marco de la crisis sanitaria por COVID-19, develó el problema estructural del sistema penitenciario, declarado como crisis carcelaria por el gobierno colombiano, ya que, según el Instituto Nacional Penitenciario y Carcelario (INPEC) para el 14 de abril habían "112 establecimientos carcelarios en el país en los que el nivel de hacinamiento supera el $20 \%$, otros 13 están por debajo de este porcentaje y apenas 7 tienen cupos disponibles"'” (El Tiempo, 2020). Esto indica que, de una capacidad de 80.928 , se atiende a una población reclusa de 120.242 personas.

Teniendo en cuenta el hacinamiento del 48 \% (González, 2020) y ante la crisis por COVID-19, el gobierno colombiano, luego de varias peticiones de la sociedad civil y sectores académicos, el 14 de abril de 2020 promulgó el Decreto 546 que buscó la excarcelación y detención domiciliaria de población recluida bajo criterios de selección determinada. El decreto buscó beneficiar a cerca de 4.000 personas en condición de reclusión, pero esa cantidad se correspondería solo con el 12 $\%$ de ese $48 \%$ de sobrepoblación la cual, al 19 de julio contaba con 1.700 casos positivos por COVID-19 (López, 2020).

Este debate no es nuevo. La Corte Constitucional de Colombia en la Sentencia T-153 de 1998 alertó por la crisis del sistema penitenciario producto del hacinamiento. Para el 2013, en la Sentencia T-388 la misma Corte se refirió a la política criminal. Indicó que era reactiva, no apegada al contexto nacional y débil en la pretensión de resocializar a la población condenada. Su invitación fue al diseño de una política no reactiva sino preventiva. Por último, en el 2015 con la Sentencia T-762 se ratifica este argumento a partir de la identificación del diseño de la 
política criminal desde la dogmática sin el acompañamiento de estudios y aportes multidisciplinarios para su diseño.

La academia colombiana no ha sido ajena a esta situación. Se ha adelantado la discusión sobre la crisis del sistema penitenciario desde varias ópticas: la de la vulneración de derechos de la población carcelaria, el hacinamiento carcelario y sus efectos, y la eficacia del sistema penitenciario para responder a la demanda de justicia del país. Iturralde (2010) plantea la precaria situación de los centros carcelarios en Colombia pese a la inversión realizada por el Estado y la relación con el aumento del encarcelamiento no por razones del incremento de los delitos, sino por un control de la criminalidad basada en patrones de coerción de un sistema penitenciario de corte autoritarios por parte del gobierno nacional. Ariza e Iturralde (2011) plantean que, pese a los intentos de reformas al sistema penitenciario en América Latina basados en la reducción del aislamiento por vía del aumento de presiones, el sistema crece, pero no mejora su eficiencia, pasando por la violación de derecho humanos y ampliando los problemas internos de las presiones.

El trabajo de Gutiérrez (2014) indaga por la realidad de la justicia penal con base en la eficacia de la pena, evidenciando las contradicciones de las funciones punitivas; Tham (2014) sustentado en la revisión de los casos de Europa, plantea el éxito que han tenido las intervenciones preventivas del crimen y la institucionalización de políticas públicas de prevención de la criminalidad; Mariño (2014) en Colombia y Durán-Chavarría (2014) para Centroamérica, proponen la necesidad de una revisión de la justicia penal para menores de edad, dando prioridad a la justicia restaurativa y el cumplimiento de la normatividad internacional como garantía de derechos de esta población; Dalto (2014) propone la necesidad de conocer el derecho indígena y aprender de las lecciones para el diseño de políticas criminales. Finalmente, Abaunza et al. (2014) dan cuenta de las difíciles condiciones de la población carcelaria de adultos mayores, la vulneración de derechos y la necesidad de crear programas de intervención a esta población. Por último, en el texto de Gutiérrez y Olarte (2018), se generan reflexiones sobre las diferentes problemáticas que se experimentan al interior de las prisiones, que atentan contra los derechos de la población carcelaria y hacen la invitación a la reflexión sobre principios normativos, su aplicabilidad y el alcance de las medidas judiciales en estas crisis.

Bajo este contexto del caso colombiano se inscribe la siguiente investigación. No indaga específicamente por el análisis del sistema penitenciario y carcelario de Colombia, pero nace de ahí y tiene la pretensión de examinar los aportes de la academia a la comprensión de los retos o desafíos de las crisis carcelarias. En el mundo, aunque con mayor evidencia en América Latina, el hacinamiento en las cárceles es un problema extremadamente grave, ya que no solo está relacionado con el sufrimiento de las personas privadas de la libertad y la vulneración de sus derechos, sino con un problema latente: las crisis de los sistemas penitenciarios y las respuestas efectivas de la política criminal de cada Estado. 
Las crisis de los sistemas penitenciarios que enfrenta el mundo, donde se incluye el hacinamiento, la sobrepoblación, la vulneración de derechos humanos al interior de las cárceles y la poca capacidad de la infraestructura penitenciaria son realidades irrefutables. Sin embargo, la mirada al tema no se agota ahí. El debate se ha centrado también en el rol de los Estados en cuanto a la atención a estas crisis. preguntas como: ¿Es efectiva la política criminal que siguen los países en crisis? ¿Qué arreglos institucionales deben hacer los Estados para atender estas crisis? Dan cuenta de ello.

El intento por responder a estos interrogantes ha guiado el diseño metodológico de esta revisión sistemática. En la búsqueda de criterios para iniciar el análisis, una revisión preliminar de los temas tratados por la academia sobre crisis carcelaria, política criminal y hacinamiento permite advertir la hoja de ruta de la revisión.

Figura 1. Concurrencia de temas consultados.

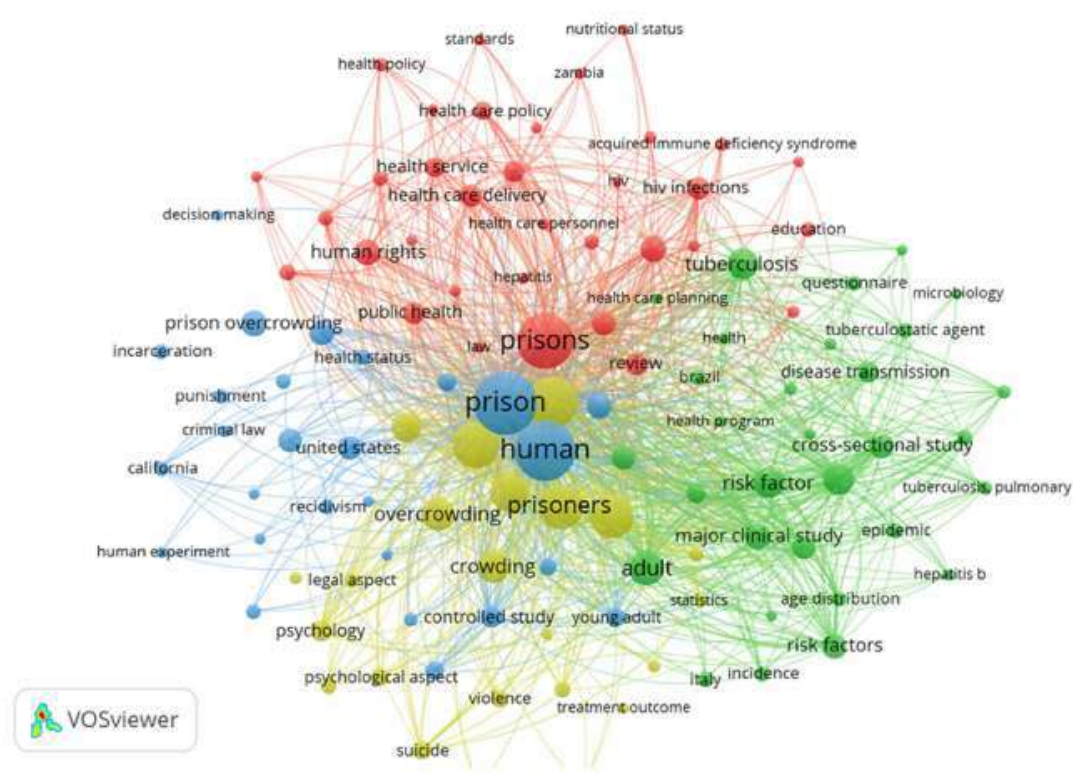

Fuente: Adaptada de Scopus (2020) VOSviewer.

La figura 1 es una red semántica de sentido construida en la primera revisión bibliográfica, que da cuenta de las conexiones entre los tres temas y permite identificar los criterios de búsqueda de la información. Tres temas de investigación se lograron reconocer: las situaciones dentro de los centros penitenciarios, las reformas propuestas a las políticas criminales y las oportunidades de mejora de los sistemas carcelarios. En este sentido, el objetivo de este artículo de revisión 
es indagar por estos debates e identificar los aportes que han realizado diversas investigaciones al dilema en el que se enfrentan los Estados para dar respuesta a la crisis carcelaria que experimentan: la reducción del hacinamiento carcelario y la eficiencia de la política criminal en materia de efectividad de los sistemas penitenciarios y la garantía de derechos humanos en los centros de reclusión.

\section{Metodología}

Para darle alcance al objetivo, se realizó una búsqueda bibliográfica en la base de datos Scopus utilizando dos palabras clave: 1 . criminal policy y 2. prison overcrowding. En la figura 1 , en la que se presenta el mapa de calor realizado con VOSviewer, se observan estas dos palabras y sus relaciones conceptuales más inmediatas y frecuentes.

Figura 2. Análisis de palabras clave.

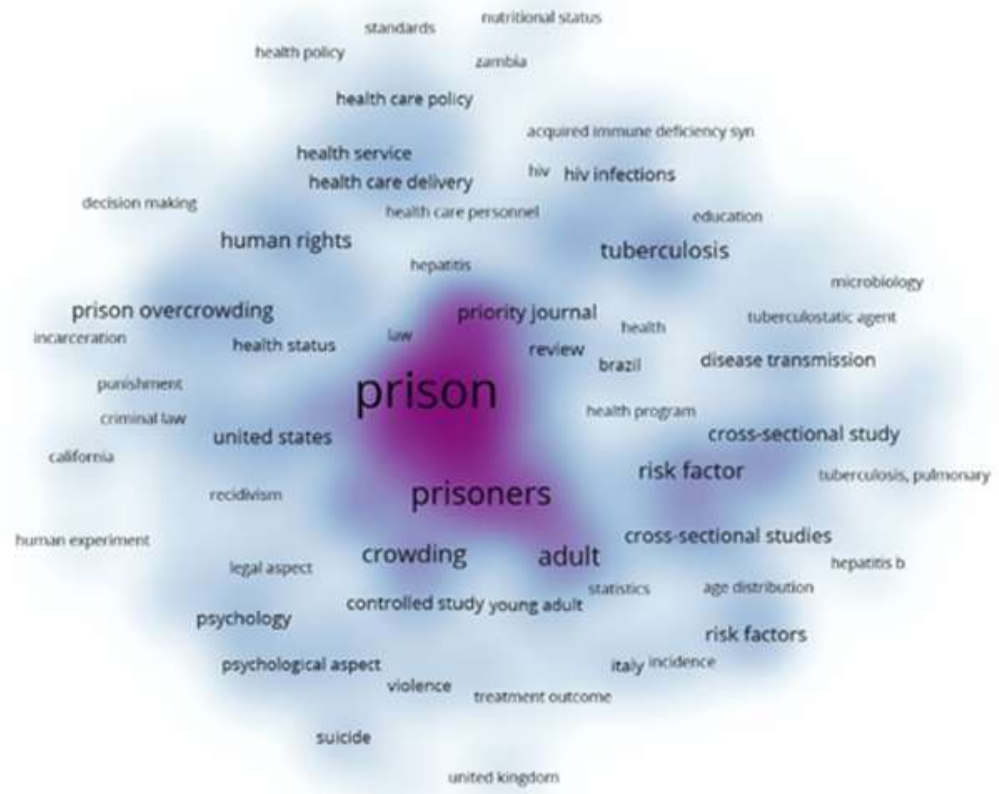

Fuente: Adaptada de Scopus (2020) VOSviewer.

La pesquisa arrojó un total de 393 documentos y se procedió a limitar la selección de referencias bibliográficas a partir de tres criterios. Primero, un período de referencia 1999-2019, que concentró el 79,4 \% de la producción académica; 
conviene decir que existen referencias desde 1959. Como resultado de esta restricción, la nueva búsqueda redujo la consulta a 249 textos. Segundo, el área de conocimiento pertinente para la investigación. Si bien Scopus identifica diecinueve áreas de conocimiento, se seleccionaron solo los textos clasificados en ciencias sociales. Esta consulta tuvo como resultado 206 textos. Tercero, se consideraron los textos más citados y aquellos que tuvieran relación directa con los criterios de la investigación, ya que subáreas como contabilidad, administración, psicología y afines estaban incluidas en el reporte. De este modo, luego de la exclusión, se trabajaron 78 textos.

Una vez identificados, se procedió a sistematizarlos en una matriz de análisis diseñada en Microsoft Word para tal fin. El análisis se desarrolló luego de la lectura correspondiente a los criterios de resultados de cada investigación.

\section{Resultados}

Producto del análisis de los textos, nace la siguiente codificación, como modelo de interpretación de los resultados de la revisión, a partir de tres categorías de análisis: 1) desafíos del sistema carcelario y la política criminal, 2) la situación actual del sistema carcelario y el hacinamiento; y, por último, 3) las opciones de respuesta al sistema carcelario. Cada una de ellas cuenta con códigos los cuales permitieron el diseño del siguiente modelo explicativo:

Figura 3. Categorías de análisis de información

Desafios

Situación actual

Opciones de respuesta
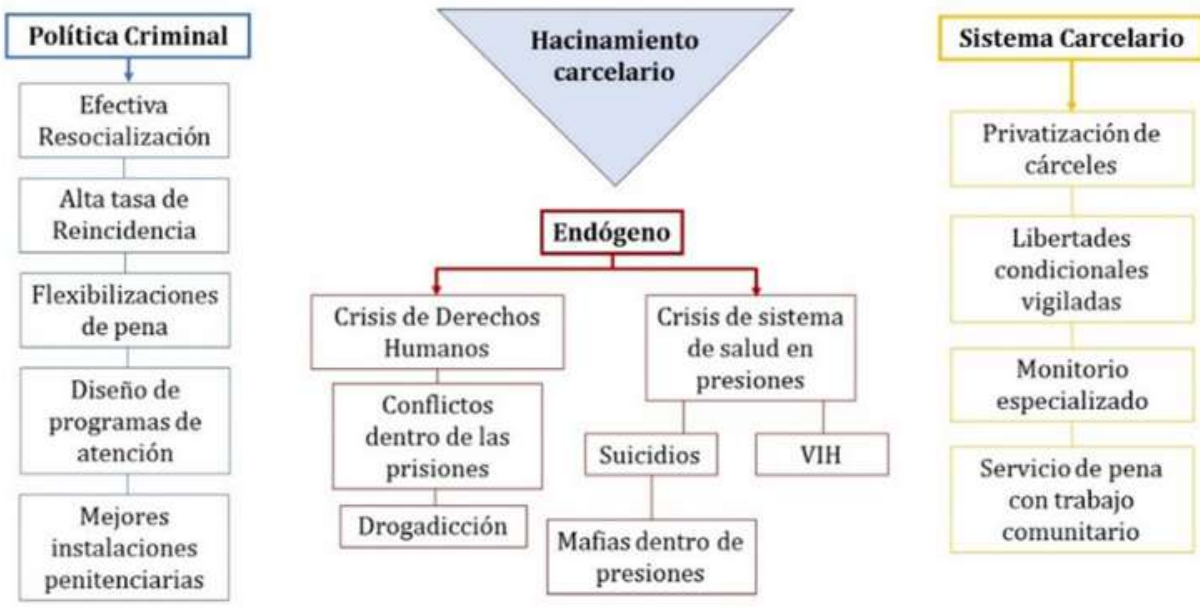

Fuente: Adaptada de Scopus (2020). 
Como se observa en la figura 3, la producción académica agrupada en las tres categorías intenta desagregarse en códigos que incluyen temas específicos que han sido abordados por las investigaciones consultadas. La primera categoría se denomina "desafíos de la política criminal" y agrupa a aquellos textos que dan cuenta de los retos en materia de resocialización, el diseño de programas penitenciarios efectivos, el control a la reincidencia, el debate alrededor de la flexibilización punitiva y la privatización carcelaria. La segunda categoría se llama "factores endógenos de las crisis carcelarias" y devela los problemas estructurales estudiados dentro de las cárceles, y finalmente, la tercera da cuenta de las propuestas, los desafíos y retos del sistema de la política criminal y los aportes desde la academia al debate por la eficiencia del sistema carcelario.

\section{Los desafíos de las políticas criminales}

Al hablar de desafíos de las políticas criminales, el primer tema que se logra evidenciar está relacionado con la propuesta de recategorización de las penas. Autores como Daems (2008), para el caso belga; o Lurigio \& Loose (2008), Alan (2011) y Barati (2019) en Estados Unidos, hacen esta invitación luego de investigar sobre el hacinamiento carcelario y la discusión alrededor de la compatibilidad de las penas y las víctimas de los casos. Bouagga (2014), Dubois \& Vrancken (2015) y Schartmueller (2015) por su parte, advierten en la necesidad de eliminar la privación y el exceso en el uso de las cadenas perpetuas. Todas estas investigaciones concluyen que, si las políticas criminales logran un equilibrio en la discusión sobre las penas, el hacinamiento se podría reducir. Sin embargo, es evidente que no solo con la relaboración punitiva se reduciría la sobrepoblación de presos, pues una modificación al sistema carcelario sin considerar la reincidencia como un factor determinante en esta sobrepoblación, genera expectativa en cualquier discusión de reforma a políticas criminales o penales.

Sobre la reincidencia, las investigaciones de Kensey \& Tournier (1999) y Nishi (2019) en Estados Unidos; y Bales, Burkes, Scaggs \& Clark (2015), Marion (2002) y Drago, Galbiati \& Vertova (2011) indagan por las estrategias de programas que buscan la reducción de la reincidencia en Europa, en especial en Francia e Italia, luego de analizar las causas de los altos casos de reincidencia producto de las fallidas políticas de intervención carcelaria y resocialización. Sobre esta última, quizá la categoría más sensible, que permite evaluar la eficacia de las políticas carcelarias, las investigaciones de Snyder, Van Wormer, Chadha \& Jaggers (2009), Johnson (2009); Dünkel, Pruin, Storgaard \& Weber (2018), Tulkens (2014), Jiménez (2017) y García \& Domínguez (2016) coinciden en la necesidad de evaluar, reformular y proponer mecanismos de seguimiento a los programas de resocialización y reasentamiento, considerando los múltiples contextos de la población 
carcelaria y la inyección de recursos fiscales para ampliar la cobertura de esta atención; pues sin una eficiente resocialización no habría finalidad de justicia de las penas impuestas.

Algunos ejemplos de análisis de estrategias para mejorar las condiciones de resocialización son el fomento a los programas educativos con énfasis en aprendizajes para la vida (Rangel, 2019) y el fortalecimiento de entornos correctivos (Brown \& Marusek, 2014) apoyados en prácticas de sociabilidad comunitaria apegados a prácticas de comunidades hawaianas , así como la propuesta de cuidado y el compromiso de trabajo colaborativo entre población penitenciaria en Inglaterra (Stewart, 2018), y el fortalecimiento de los lazos de solidaridad entre la población carcelaria, a partir de las condiciones socioeconómicas similares, adelantados en Italia (Santorso, 2015).

Finalmente, se advierte una línea de investigación que atina a proponer, como mecanismo para la reducción de la crisis de sobrepoblación carcelaria y la garantía de no vulneración de derechos, la privatización de los centros penitenciarios. A partir del caso israelí, Timor (2006); Schabbach (2020) para el brasilero y Price \& Riccucci (2005) en Estados Unidos, se explora la eficacia en la reducción de las crisis de hacinamiento y conductas violentas luego de la implementación de administraciones militares o privadas de estos centros carcelarios. Esto, bajo la hipótesis de que la sobrepoblación, reincidencia y el crimen son producto de la misma lógica endógena de las prisiones y de la debilidad institucional para intervenir estos estos casos dentro de las mismas.

\section{La crisis carcelaria y el hacinamiento: el problema endógeno}

El anterior argumento, si bien es producto de los resultados de las investigaciones y se propone como una alternativa a debatir, permite develar los problemas endógenos que viven los centros de reclusión y que han llamado la atención de la comunidad académica. Se ha denominado endógeno siguiendo el argumento de Glazener \& Nakamura (2020), quienes advierten la fuerte relación entre sobrepoblación y mala conducta dentro de los centros penitenciarios.

Siguiendo este argumento, el tema de condiciones violentas y su relación con el hacinamiento dentro de las cárceles es estudiado, en particular para América Latina, por Cabanillas (2012), Krüger, de Oliveira \& Mariani (2018) y Limoncelli, Mellow \& Na (2020). Para Italia, por Ricciardelli, Power \& Medeiros (2018). Y para Estados Unidos, por Lurigio \& Lurigio (2016), Guetzkow \& Schoon (2015), y Erasmus \& Hornigold (2015) y Paoline \& Lambert (2012), quienes evidencian prácticas de violencia tanto de población penitenciaria, relacionadas con peleas entre grupos por control territorial de las cárceles, consumo de sustancias psicoactivas, 
así como también casos de violencia infringida por parte de actores institucionales. Estas prácticas, sumadas al contexto de sobrepoblación, se presentan como los retos susceptibles de intervención más inmediatos debido a la posible violación de Derechos Humanos, como lo evidencian en América Latina Matthews (2011) y Ungar \& Magaloni (2009); y la posibilidad de generar conflictos endógenos de mayores proporciones.

La salud es un tema recurrente cuando se investiga por la crisis del hacinamiento carcelario. La salud mental, y en especial el suicidio, ocupa buena parte de la atención de las investigaciones revisadas. Estudios como los de Fritz et al. (2020) que advierten de los 1.324 suicidios en diez cárceles en América Latina. 0 van Ginneken, Sutherland \& Molleman (2017) quienes analizan los casos en Inglaterra y Gales producto del hacinamiento; o Preti \& Cascio (2006) y Franklin, Franklin \& Pratt (2006) quienes dan cuenta de los comportamientos autolesivos producto de las crisis internas de las penitenciarías en Italia; o Duthé, Hazard, Kensey \& Ké Shon (2009) en Francia y Rabe (2012) y Dye (2010) en Estados Unidos, por citar algunos ejemplos, coinciden en determinar no solo la necesidad de intervenir este fenómeno de manera médica sino también social. Programas de intervención psicosocial, ecológicos y de prevalencia, según las investigaciones, permitirían disminuir la incidencia de estos casos, así como determinar el acompañamiento y seguimiento a los casos de ideación suicida recurrente en las penitenciarías.

Por su parte, y continuando con los estudios sobre salud y hacinamiento carcelario, investigaciones como las de Hough, Allen \& Solomon (2008), Spencer (2012) y Elger, Ritter \& Stöver (2016) en Estados Unidos; Barsaglini, Kehrig \& de Arruda (2015) en Brasil; y Sander \& Lines (2016) en América Latina, advierten de las difíciles condiciones en las que vive la población carcelaria producto de la deficiencia en el sistema de atención en salud. Casos como VIH, hepatitis y tuberculosis son afecciones recurrentes y de difícil detección, con alta propagación debido a la sobrepoblación, el hacinamiento y los débiles sistemas de salud penitenciarios existentes.

\section{Las opciones de respuesta al sistema carcelario}

Considerando los problemas endógenos que se han identificado en las referencias bibliográficas consultadas, conviene iniciar señalando las estrategias que se proponen como opciones de respuesta al último de los problemas expuestos: el de salud, toda vez que, es un imperativo luego que el COVID-19 afectara también las prisiones del mundo. Ante estas situaciones, la excarcelación inteligente o liberación compasiva se ha propuesto como una solución al hacinamiento y a la reducción de costos de tratamientos de enfermedades crónicas de salud de la población penitenciaria. 
Estados Unidos es quizá el contexto donde más referencias aparecen frente a esta alternativa. Las investigaciones de Pitts, Griffin \& Johnson (2014), Sundt, Salisbury \& Harmon (2016), Skinns (2016) y Holland, Prost, Hoffmann \& Dickinson (2020), y Alexander, Allo \& Klukoff (2020), han evaluado las iniciativas de liberación inteligente y compasiva de la población que presenta dificultad en sus condiciones de salud o enfermedades terminales. Igual conclusión se evidencian en los textos de Handtke, Wangmo, Elger \& Bretschneider (2017) analizando esta circunstancia en Europa; Nunes (2015) en Brasil; Zevallos (2016) en Perú, y Skinns (2016) en América Latina.

Muy similar a esta propuesta, pero considerando no solo asuntos de salud o argumentos compasivos, sino la excarcelación por delitos menores, las investigaciones de Herzog-Evans (2019) en Francia y la propuesta de lanzamiento anticipado, Losier (2017) y el análisis de la ley y el orden en New York o Simon (2017) sobre la política de enjuiciamiento en Estados Unidos, los debates de la legislación belga evidenciados por Cliquennois \& Herzog-Evans (2018) y la revisión de los casos de delitos menores en India adelantada por Saxena (2020), ponen sobre la mesa una propuesta al sistema carcelario ambiciosa como polémica, toda vez que, implicaría una revisión exhaustiva de los marcos normativos y penales y la reconfiguración de estrategias de seguimiento a la población que llegase a ser beneficiada, en procura de limitar la reincidencia en casos de sensible atención.

Sin duda, resulta una propuesta muy polémica, y más en medio de una pandemia, pero el control y seguimiento a estos casos es un desafío, y ante ello, la propuesta del monitoreo activo se convierte en una posible solución anticipada a esta situación. La gestión de estas alternativas de monitoreo es estudiada por Herzog-Evans (2020) en Brasil; así como también por Dünkel, Thiele \& Treig (2017) en Alemania; Maes, Mine De Man \& Van Brakel (2012); Maes \& Mine (2013) y Beyens \& Roosen (2017) en Bélgica; Simon (2018) en Estados Unidos, ven en los casos de monitoreo electrónico una medida eficaz para resolver los problemas de hacinamiento carcelario.

La última de las alternativas propuestas e identificadas tiene que ver con el fomento a la detención domiciliaria o la liberación condicional, e incluso de cumplimiento de las penas a partir de trabajo comunitario. Estas conclusiones se encuentran en las investigaciones de Plesničar (2012) y Meško, Fields \& Smole (2011) para la revisión del caso en Eslovenia; en China la indagación de Jiang et al. (2014); Bonta \& Gendreau (2019) y Zanis et al. (2003) en Estados Unidos; Di Vita (2020) y Gualazzi, Mancuso, \& Mangiaracina (2012) en Italia; Maxfield \& Baumer (1992) y Anagnostaki (2011) en Grecia. Todas estas investigaciones tienen un punto de inflexión en su análisis: propenden por la implementación de medidas. Si bien, estas alternativas pueden leerse como propuestas de reforma al sistema penitenciario sobre la base de atenciones efectivas desde la inmediatez de las crisis, giran alrededor de un diseño institucional que abogue por la prevención 
de los delitos como solución final a los problemas que las crisis penitenciales develan: la garantía de derechos, la reducción del hacinamiento y la resocialización efectiva de la población condenada.

\section{Conclusiones}

El hacinamiento en un fenómeno carcelario y una crisis por sí mismo, ya que es la cabeza de turco que devela problemas estructurales dentro de los centros de reclusión. Dentro de las cárceles, la problemática se puede explicar en tres vías. La primera, está relacionada con aquellos factores endógenos que complejizan las condiciones de vida de la población recluida. Las violencias vividas dentro de las cárceles por el control territorial, el consumo de sustancias psicoactivas y las riñas son factores que no son solo poco estudiados, sino poco intervenidos por la institucionalidad. La segunda vía está relacionada con las demandas por la garantía de derechos humanos. Casos de abusos de fuerza, violaciones a derechos y tratos indebidos han sido denunciados en no pocas oportunidades. La tercera está relacionada con la salud integral y la salud mental de la población recluida. Casos de pacientes con enfermedades crónicas sin atender, población sometida a contagios y el suicidio, son problemas que han sido intervenidos con mucha debilidad por los gobiernos.

Como soluciones a estos problemas, se advierte en la bibliografía consultada al menos cuatro propuestas o desafíos al sistema carcelario. El primero de ellos está relacionado con las propuestas por la reducción del hacinamiento por vía de la excarcelación inteligente o liberación compasiva, más usada en contextos de crisis de salud, como la vivida por la pandemia de COVID-19. El segundo, quizá el que más polémica puede generar, propugna por la efectividad de una reforma a la ponderación de la severidad de las penas. El tercero, se refiere a la reducción de la sobrepoblación por vía de la excarcelación monitoreada, no solo a través de dispositivos electrónicos, sino por mecanismos sociales de trabajo comunitario y, la última, por la reclusión domiciliaria. Estas dos últimas, son empleadas en muchos países y se encuentran en constante evaluación de eficacia.

Todas las propuestas, están soportadas en amplias y relevantes investigaciones en cada uno de los países de consulta. En América Latina, el desafío aún está latente. Implementar una o varias de las alternativas rastreadas, implica un rediseño institucional a la política criminal y a los sistemas penitenciarios, que, dicho sea de paso, urgen por soluciones efectivas a las crisis que cada día develan la fragilidad de la resocialización de la población carcelaria y la garantía de justicia plena de la sociedad. En suma, estas apuestas parecen ser de corte inmediato a las crisis, pero en esencia, conservan el espíritu de reforma institucional que da paso a la prevención de los delitos. 


\section{Referencias bibliográficas}

Abaunza, C.I., Bustos, P., Enríquez, K., Mendoza, M, Molina., Padilla, A. y Paredes, G. (2014). Adulto mayor: prisión y sociedad. En Gutiérrez, M. et al. (eds.), Política Criminal y Libertad (pp. 271-306). Bogotá: Universidad Externado de Colombia. Recuperado de https://books.openedition.org/uec/1068

Alan, H. (2011). Prison overcrowding Corrections. Thousand Oaks, CA: SAGE Publications. Retrieved from https://www.scopus.com/inward/record.uri?eid=2-s2.084950128850\&doi=10.4135\%2f9781412994101.n15\&partnerID=40\&md5=

Alexander, A., Allo, H., Klukoff, H. (2020). Sick and Shut In: Incarceration During a Public Health Crisis. Journal of Humanistic Psychology. Retrieved from https:// www.scopus.com/inward/record.uri?eid=2-s2.0-85086122869\&doi=10.1177 \%2f0022167820930556\&partnerID=40\&md5=

Anagnostaki, M. (2011). Community service in greece: Sentencing practices, the role of the prosecution service and local authorities. European Journal of Criminology, 8(2), 157-166. Retrieved from https://www.scopus.com/inward/record. uri?eid=2-s2.0-79952597083\&doi=10.1177\%2f1477370810397256\&partne $\mathrm{rID}=40 \& \mathrm{md} 5=$

Ariza, L. e Iturralde, M. (2011). Los muros de la infamia: prisiones en Colombia y en América Latina. Bogotá: Universidad de los Andes, Facultad de Derecho, CIJUS, Ediciones Uniandes.

Bales, W., Burkes, K., Scaggs, S. \& Clark, C. (2015). Recidivism International Encyclopedia of the Social \&amp. Behavioral Sciences (Second Edition). Retrieved from https://www.scopus.com/inward/record.uri?eid=2-s2.085043441266\&doi=10.1016\%2fB978-0-08-097086-8.45079-8\&partnerID=

Barati, M. (2019). Punishment Severity and Crime: The Case of Arkansas. Review of Law and Economics, 15(1). Retrieved from https://www.scopus.com/inward/ record.uri?eid=2-s2.0-85064837060\&doi=10.1515\%2frle-2017-0025\&partne rID=40\&md5=4c8aa8207cdded31130faa91c9bb95bd

Barsaglini, R., Kehrig, R. \& de Arruda, M. (2015). Analysis of the perception of managers on management of prison health policy in Mato Grosso, Brazil. Saude e Sociedade, 24(4), 1119-1136. Recuperado de https://www.scopus.com/inward/record. uri?eid=2-s2.0-84982732303\&doi=10.1590\%2fS0104-12902015138278\&pa rtnerID $=40 \& \mathrm{md} 5=$

Beyens, K. \& Roosen, M. (2017). Electronic monitoring and reintegration in Belgium. European Journal of Probation, 9(1), 11-27. Retrieved from https://www.scopus. com/inward/record.uri?eid=2-s2.0-85045343481\&doi=10.1177\%2f20662203 $17704132 \&$ partnerID $=40 \& \mathrm{md} 5=$ 
Bonta, J. \& Gendreau, P. (2019). Coping with prison Psychology and Social Policy. Retrieved from https://www.scopus.com/inward/record.uri?eid=2-s2.085079316841\&doi=10.4324\%2f9781315793030-26\&partnerID=40\&md5=

Bouagga, Y. (2014). Time to punish: Manage waiting in remand. Terrain, 63, 86-101. Retrieved from https://www.scopus.com/inward/record.uri?eid=2-s2.084906901674\&doi=10.4000\%2fterrain.15508\&partnerID=40\&md5=375f56 d315c60468ed411ac792c41fde

Brown, M. \& Marusek, S. (2014). 'Ohana Ho'opakele: The Politics of Place in Corrective Environments. International Journal for the Semiotics of Law, 27(2), 225-242. Retrieved from https://www.scopus.com/inward/record.uri?eid=2-s2.084900790068\&doi=10.1007\%2fs11196-012-9291-8\&partnerID=40\&md5=

Cabanillas, O. (2012). Violent Conflict and Prison Overcrowding. Desarrolloy Sociedad, (69), 33-71. Retrieved from https://www.scopus.com/inward/record.uri?eid=2s2.0-84863533582\&partnerID=40\&md5=7ad85f18eec17faf8ede61219a8ea0c8

Cliquennois, G. \& Herzog-Evans, M. (2018). European monitoring of Belgian and French penal and prison policies Crime. Law and Social Change, 70(1), 113134. Retrieved from https://www.scopus.com/inward/record.uri?eid=2-s2.085034211638\&doi=10.1007\%2fs10611-017-9722-2\&partnerID=40\&md5=

Coyle, A., Heard, C. \& Fair, H. (2016). Current trends and practices in the use of imprisonment. International Review of the Red Cross, 98(903), 761-781. Retrieved from https://www.scopus.com/inward/record.uri?eid=2-s2.0-85041345458\&doi=1 $0.1017 \% 2 f S 1816383117000662 \&$ partnerID=40\&md5=

Daems, T. (2008). ¿Compatible victims? Prison overcrowding and penal reform in Belgium. International Journal of Law, Crime and Justice, 36(3), 153-167. Retrieved from https://www.scopus.com/inward/record.uri?eid=2-s2.0$55149114663 \&$ doi=10.1016\%2fj.ijlcj.2008.04.001\&partnerID=40\&md5=4f9 e8dcdb94ebcbdd73de0ea0c292c68

Dalto, M. (2014). El aporte del derecho indígena a la construcción de las políticas criminales. En Gutiérrez, M. et al. (eds.), Política Criminal y Libertad (pp. 239-269). Bogotá: Universidad Externado de Colombia.

Di Vita, G. (2020). Recent legislative measures to reduce overcrowding of prisons in Italy: a preliminary assessment of their economic impact. European Journal of Law and Economics, 49(2), 277-299. Retrieved from https://www.scopus.com/ inward/record.uri?eid=2-s2.0-85078204684\&doi=10.1007\%2fs10657-02009639-5\&partnerID=40\&md5=

Drago, F., Galbiati, R. \& Vertova, P. (2011). Prison Conditions and Recidivism. American Law and Economics Review, 13(1), 103-130. Retrieved from https://www.scopus. com/inward/record.uri?eid=2-s2.0-79959715531\&doi=10.1093\%2faler\%2fah q024\&partnerID=40\&md5=05164cb6d1e14970a602fa44eb52c432 
Dubois, C. \& Vrancken, D. (2015). Restorative detention or 'work on self'? Two accounts of a Belgian prison policy. Ethnography, 16(2), 187-206. Retrieved from https:// www.scopus.com/inward/record.uri?eid=2-s2.0-84930450957\&doi=10.1177 \%2f1466138114538803\&partnerID=40\&md5=

Dünkel, F., Pruin, I., Storgaard, A. \& Weber, J. (2018). Comparable aims and different approaches; Prisoner resettlement in Europe - concluding remarks Prisoner Resettlement in Europe. Recuperado de https://www.scopus.com/inward/ record.uri?eid=2-s2.0-85077345582\&doi=10.4324\%2f978131519459227\&partnerID $=40 \& \mathrm{md} 5=$

Dünkel, F., Thiele, C. \& Treig, J. (2017). “You'll never stand-alone”: Electronic monitoring in Germany. European Journal of Probation, 9(1), 28-45. Retrieved from https:// www.scopus.com/inward/record.uri?eid=2-s2.0-85045311498\&doi=10.1177 \%2f2066220317697657\&partnerID=40\&md5=

Durán-Chavarría, D. (2014). Derecho penal juvenil en América central: avances y riesgos. En Gutiérrez, M. et al. (eds.), Política Criminal y Libertad (pp. 215-237). Bogotá: Universidad Externado de Colombia. Recuperado de https://books. openedition.org/uec/1058

Duthé, G., Hazard, A., Kensey, A. \& Ké Shon, J. (2009). Suicide in prison: A comparison between France and its European neighbours. Population and Societies, (462), 1-4. Retrieved from https://www.scopus.com/inward/ record.uri?eid=2-s2.0-77955034241\&partnerID $=40 \& \mathrm{md} 5=5698 \mathrm{a} 37 \mathrm{f} 4 \mathrm{~d}$ 2de1f314adf369bf218035

Dye, M. (2010). Deprivation, importation, and prison suicide: Combined effects of institutional conditions and inmate composition. Journal of Criminal Justice, 38(4), 96-806. Retrieved from https://www.scopus.com/inward/record. uri?eid=2-s2.0-77954759577\&doi=10.1016\%2fj.jcrimjus.2010.05.007\&partn erID=40\&md5=

El Tiempo. (14 de abril de 2020). Estas son las 5 cárceles con mayor nivel de hacinamiento del país. El Tiempo. Recuperado de https://www.eltiempo.com/justicia/ investigacion/estas-son-las-carceles-mas-hacinadas-de-colombia-484076

Elger, B., Ritter, C. \& Stöver, H. (2016). Emerging issues in prison health Emerging Issues in Prison Health, 1-259. Retrieved from https://www.scopus.com/inward/ record.uri?eid=2-s2.0-85068843238\&doi=10.1007\%2f978-94-017-75588\&partnerID=40\&md5=

Erasmus, D. \& Hornigold, A. (2015). Court supervised institutional transformation in South Africa. Potchefstroom Electronic Law Journal, 18(7), 2457-2501. Retrieved from https://www.scopus.com/inward/record.uri?eid=2-s2.085037712943\&doi=10.4314\%2fpelj.v18i7.02\&partnerID $=40 \& \mathrm{md5}=1026 \mathrm{cec}$ 3c4979f7aa6afec88e0e1be3c 
Franklin, T., Franklin, C. \& Pratt, T. (2006). Examining the empirical relationship between prison crowding and inmate misconduct: A meta-analysis of conflicting research results. Journal of Criminal Justice, 34(4), 401-412. Retrieved from https://www. scopus.com/inward/record.uri?eid=2-s2.0-33748432541\&doi=10.1016\%2fj.jc rimjus.2006.05.006\&partnerID $=40 \& \mathrm{md} 5=$

Fritz, F., Fazel, S., Benavides, A., Henry, P., Rivera, G., Torales, J.,... \& Mundt, A. (2020). 1324 prison suicides in 10 countries in South America: incidence, relative risks, and ecological factors Social Psychiatry and Psychiatric Epidemiology. Retrieved from https://www.scopus.com/inward/ record.uri?eid=2-s2.0-85084598569\&doi=10.1007\%2fs00127-020-01871$3 \&$ partnerID $=40 \& \mathrm{md} 5=$

García, L. \& Domínguez, A. (2016). The reality of the penitentiary system in Colombia: The need to introduce a new criminal policy. Revista Criminalidad, 58(2), 175195. Retrieved from https://www.scopus.com/inward/record.uri?eid=2-s2.085018969376\&partnerID=40\&md5=b91344b9d5564db92a076f92add8aceb

Glazener, E. \& Nakamura, K. (2020). Examining the Link Between Prison Crowding and Inmate Misconduct: Evidence from Prison-Level Panel Data. Justice Quarterly,37(1), 109-131. Retrieved from https://www.scopus.com/inward/record. uri?eid=2-s2.0-85055696640\&doi=10.1080\%2f07418825.2018.1495251\&par therID $=40$ \&

González, L. (2020). La Crisis carcelaria en el marco del Covid 19. nodal. Recuperado de https://www.nodal.am/2020/05/colombia-la-crisis-carcelaria-en-el-marcodel-covid-19-por-leonardo-gonzalez-p/\#_ftn1

Gualazzi, A., Mancuso, C. \& Mangiaracina, A. (2012). 'Back Door Sentencing' in Italy: Common Reasons and Main Consequences for the Recall of Prisoners European. Journal of Probation, 4(1), 73-84. Retrieved from https://www.scopus.com/ inward/record.uri?eid=2-s2.0-84988923616\&doi=10.1177\%2f20662203120 0400107\&partnerID=40\&md5=

Guetzkow, J. \& Schoon, E. (2015). If You Build It, They Will Fill It: The Consequences of Prison Overcrowding. Litigation Law and Society Review, 49(2), 401-432. Retrieved from https://www.scopus.com/inward/record.uri?eid=2s2.0-84929145882\&doi=10.1111\%2flasr.12140\&partnerID=40\&md5=d5367 88dadb89ba6b7927da2fa7743a6

Gutiérrez, M. (2014). Hacia una justicia restaurativa. En Gutiérrez, M. et al. (eds.), Política Criminal y Libertad (pp. 71-90). Bogotá: Universidad Externado de Colombia. Recuperado de https://books.openedition.org/uec/1030

Gutiérrez, M., Tham, H., Mariño, C., Durán-Chavarría, D., Dalto, M., Abaunza, C.,... y Paredes, G. (2014). Política Criminal y Libertad. Bogotá: Universidad Externado de Colombia. 
Gutiérrez, M. y Olarte, A. (2018). Los riesgos del puntivismo, presunción de inocencia e indignidad carcelaria en Colombia. Bogotá: Universidad Externado de Colombia.

Handtke, V., Wangmo, T., Elger, B. \& Bretschneider, W. (2017). New guidance for an old problem: Early release for seriously ill and elderly prisoners in Europe. Prison Journal, 97(2), 224-246. Retrieved from https://www.scopus.com/inward/ record.uri?eid $=2$-s2.0-85018292575\&doi $=10.1177 \% 2 f 0032885517692805 \&$ partnerID $=40 \&$ md5 $=$

Herzog-Evans, M. (2019). French early release: McProcedures and McRe-entry. European Journal of Probation, 11(3), 188-201. Retrieved from https://www.scopus. com/inward/record.uri?eid=2-s2.0-85077337726\&doi=10.1177\%2f20662203 19897238\&partnerID $=40 \& \mathrm{md} 5=$

Herzog-Evans, M. (2020). French Prison Day Leave and the Rationale Behind It: Resocialisation or Prison Management?. European Journal on Criminal Policy and Research. Retrieved from https://www.scopus.com/inward/ record.uri?eid=2-s2.0-85085901061\&doi=10.1007\%2fs10610-020-094454\&partnerID $=40$ \&md $5=$

Holland, M., Prost, S, Hoffmann, H. \& Dickinson, G. (2020). Access and utilization of compassionate release in state departments of corrections Mortality. Retrieved from https://www.scopus.com/inward/record.uri?eid=2-s2.0-85083565039\&doi= 10.1080\%2f13576275.2020.1750357\&partnerID $=40 \&$

Hough, M., Allen, R. \& Solomon, E. (2008). Tackling prison overcrowding: Build more prisons? sentence fewer offenders? Policy Press. Retrieved from https://www. scopus.com/inward/record.uri?eid=2-s2.0-85033338265\&partnerID=40\&m d5=0ba9537574019ddcf2a706e29f6bc639

Iturralde, M. (2010). Castigo, liberalismo autoritario y justicia penal de excepción. Bogotá: Siglo del Hombre Editores, Universidad de los Andes, Instituto Pensar, Pontificia Universidad Javeriana.

Jiang, S., Jiang, S., Xiang, D., Chen, Q., Huang, C., Yang, S.,... \& Zhao, A. (2014). Community Corrections in China: Development and Challenges. The Prison Journal, 94(1), 75-96. Retrieved from https://www.scopus.com/inward/record.uri?eid=2-s2.084893454650\&doi=10.1177\%2f0032885513512091\&partnerID=40\&md5=

Jiménez, N. (2017). Resocialization as end of sentence - A frustration with the Colombian penitentiary and prison system. Caderno CRH, 30(81), 539-559. Retrieved from https://www.scopus.com/inward/record.uri?eid=2-s2.085038429397\&doi=10.1590\%2fS0103-49792017000300010\&partnerID=40\&

Johnson, J. (2009). Lifetech institute: Leading change through transitional centers. Community College Journal of Research and Practice, 33(11), 942-944. Retrieved from https://www.scopus.com/inward/record.uri?eid=2-s2.0-85011468705\&doi=1 $0.1080 \% 2 f 10668920903153501 \&$ partnerID=40\&md5= 
Kensey, A. \& Tournier, P. (1999). Prison population inflation, overcrowding and recidivism: The situation in France. European Journal on Criminal Policy and Research, 7(1), 97-119. Retrieved from https://www.scopus.com/inward/record. uri?eid=2-s2.0-34247283330\&doi=10.1023\%2fA\%3a1008792215712\&partn erID $=40 \& \operatorname{md} 5=$

Krüger, C., de Oliveira, D. \& Mariani, M. (2018). Inside the prison: Evidence of institutional violence in a female prison on the border between Brazil and Bolivia. Dilemas, 11(3), 435-452. Retrieved from https://www.scopus.com/inward/ record.uri?eid=2-s2.0-85064011311\&partnerID=40\&md5=cd753bd810a3ea 197fecae2ceec5a40d

Limoncelli, K., Mellow, name]J. \& Na, C. (2020). Determinants of Intercountry Prison Incarceration Rates and Overcrowding in Latin America and the Caribbean. International Criminal Justice Review, 30(1), 10-29. Recuperado de https://www. scopus.com/inward/record.uri?eid=2-s2.0-85062467052\&doi=10.1177\%2f1 057567719830530\&partnerID=40\&md5 =

López, D. (9 de julio de 2020). Hacinamiento carcelario está en su punto más bajo desde el 2011. El Tiempo. Recuperado de https://www.eltiempo.com/justicia/ servicios/se-reduce-hacinamiento-carcelario-por-el-coronavirus-al-punto-masbajo-en-10-anos-515974

Losier, T. (2017). Against 'law and order' lockup: the 1970 NYC jail rebellions. Race and Class, 59(1), 3-35. Retrieved from https://www.scopus.com/inward/record. uri?eid=2-s2.0-85021937361\&doi=10.1177\%2f0306396817707431\&partner ID $=40 \& m d 5=$

Lurigio, A. \& Loose, P. (2008). The disproportionate incarceration of African Americans for drug offenses: The national and Illinois perspective. Journal of Ethnicity in Criminal Justice, 6(3), 223-247. Retrieved from https://www.scopus.com/ inward/record.uri?eid=2-s2.0-67649432601\&doi=10.1080\%2f15377930802 243445\&partnerID=40\&md5=

Lurigio, A. \& Lurigio, A. (2016). Jails in the United States: The "Old-New". Frontier in American Corrections The Prison Journal, 96(1), 3-9. Retrieves from https://www. scopus.com/inward/record.uri?eid=2-s2.0-84958191295\&doi=10.1177\%2f00 $32885515605462 \&$ partnerID $=40 \& \operatorname{md} 5=$

Maes, E. \& Mine, B. (2013). Some Reflections on the Possible Introduction of Electronic Monitoring as an Alternative to Pre-trial Detention in Belgium Howard. Journal of Criminal Justice, 52(2), 144-162. Retrieved from https://www.scopus.com/ inward/record.uri?eid=2-s2.0-84875790177\&doi=10.1111\%2fhojo.12008\&p artnerID=40\&md5=76b83e48ea5e98ce500daa134ac240c3

Maes, E., Mine, B., De Man, C. \& Van Brakel, R. (2012). Thinking about Electronic Monitoring in the Context of Pre-Trial Detention in Belgium: A Solution to Prison 
Overcrowding? European Journal of Probation, 4(2), 3-22. Retrieved from https:// www.scopus.com/inward/record.uri?eid=2-s2.0-84906037517\&doi=10.1177 $\% 2 \mathrm{f} 206622031200400202 \&$ partnerID $=40 \& \mathrm{md} 5=$

Mariño, C. (2014). Justicia juvenil restaurativa como respuesta alternativa. En Gutiérrez, M. et al. (eds.), Política Criminal y Libertad (pp. 169-214). Bogotá: Universidad Externado de Colombia. Recuperado de https://books.openedition.org/uec/1052

Marion, N. (2002). Effectiveness of Community based Correctional Programs: A Case Study. The Prison Journal, 82(4), 478-497. Retrieved from https://www.scopus. com/inward/record.uri?eid=2-s2.0-20444412549\&doi=10.1177\%2f0032885 502238682\&partnerID $=40 \& \mathrm{md} 5=$

Matthews, R. (2011). A realist proposal for prison reform in Latin America. Política Criminal, 6(12), 296-338. Retrieved from https://www.scopus.com/ inward/record.uri?eid=2-s2.0-84858630926\&doi=10.4067\%2fS071833992011000200003\&partnerID $=40 \&$

Maxfield, M. \& Baumer, T. (1992). Pretrial Home Detention with Electronic Monitoring: A Nonexperimental Salvage. Evaluation Review, 16(3), 315-332. Recuperado de https://www.scopus.com/inward/record.uri?eid=2-s2.0-84973821489\&doi= 10.1177\%2f0193841X9201600306\&partnerID=40\&md5=

Meško, G., Fields, C. \& Smole, T. (2011). A concise overview of penology and penal practice in Slovenia: The unchanged capacity, new standards, and prison overcrowding. Prison Journal, 91(4), 398-424. Retrieved from https://www.scopus. com/inward/record.uri?eid=2-s2.0-82555205379\&doi=10.1177\%2f0032885 511424389\&partnerID $=40 \& \mathrm{md} 5=$

Nishi, A. (2019). Privatizing sentencing: A delegation framework for recidivism risk assessment. Columbia Law Review, 119(6), 1671-1710. Retrieved from https:// www.scopus.com/inward/record.uri?eid=2-s2.0-85075205791\&partnerID $=4$ $0 \& \mathrm{md} 5=1 \mathrm{~d} 385 \mathrm{c} 436 \mathrm{cf} 3 \mathrm{fbcea} 4893 \mathrm{c} 324 \mathrm{~d} 3 \mathrm{f} 7 \mathrm{bcd}$

Nunes, R. (2015). The politics of sentencing reform in Brazil: Autonomous bureaucrats, constrained politicians and gradual policy change. Journal of Latin American Studies, 47(1), 121-148. Recuperado de https://www.scopus.com/inward/ record.uri?eid=2-s2.0-84921439077\&doi=10.1017\%2fS0022216X14001060 \&partnerID $=40 \& \mathrm{md} 5=$

Paoline, E. \& Lambert, E. (2012). The Issue of Control in Jail: The Effects of Professionalism, Detainee Control, and Administrative Support on Job Stress, Job Satisfaction, and Organizational Commitment among Jail Staff. American Journal of Criminal Justice, 37(2), 179-199. Retrieved from https://www.scopus.com/ inward/record.uri?eid=2-s2.0-84860519344\&doi=10.1007\%2fs12103-0119128-0\&partnerID=40\&md5= 
Pitts, J., Griffin., O. \& Johnson, W. (2014). Contemporary prison overcrowding: shortterm fixes to a perpetual problem. Contemporary Justice Review: Issues in Criminal, Social, and Restorative Justice, 17(1), 124-139. Retrieved from https://www. scopus.com/inward/record.uri?eid=2-s2.0-84897027913\&doi=10.1080\%2f1 0282580.2014.883844\&partnerID=40\&md5=

Plesničar, M. (2012). The Slovenian system of conditional reléase in light of recent trends in the field. Revija za Kriminalistiko in Kriminologijo, 63(2), 136-148. Retrieved from https://www.scopus.com/inward/record.uri?eid=2-s2.0-84894670420\&partne rID=40\&md5=ea2792d1bbbe8faa3bf46e10780afcb6

Preti, A. \& Cascio, M. (2006). Prison suicides and self-harming behaviours in Italy, 1990-2002. Medicine, Science and the Law, 46(2), 127-133. Retrieved from https://www.scopus.com/inward/record.uri?eid=2-s2.0-33646449097\&doi= 10.1258\%2frsmmsl.46.2.127\&partnerID=40\&md5=8cc1e099531c09c713f99 $955 b f a a 7146$

Price, B. \& Riccucci, N. (2005). Exploring the determinants of decisions to privatize state prisons. American Review of Public Administration, 35(3), 223-235. Retrieved from https://www.scopus.com/inward/record.uri?eid=2-s2.0-24944484357\&doi=1 0.1177\%2f0275074005277174\&partnerID=40\&md5=

Rabe, K. (2012). Prison structure, inmate mortality and suicide risk in Europe. International Journal of Law and Psychiatry, 35(3), 222-230. Retrieved from https://www. scopus.com/inward/record.uri?eid=2-s2.0-84859833133\&doi=10.1016\%2fj.ij lp.2012.02.012\&partnerID=40\&md5=5ed55d3d580f6c0c48a2c10f27c7b31c

Rangel, H. (2019). Cooperation and education in prison: A policy against the tide in the Latin American penitentiary crisis. International Review of Education, 65(5), 785809. Retrieved from https://www.scopus.com/inward/record.uri?eid=2-s2.085055993454\&doi=10.1007\%2fs11159-018-9747-5\&partnerID=40\&md5=

Ricciardelli, R., Power, N. \& Medeiros, D. (2018). Correctional Officers in Canada: Interpreting Workplace. Violence Criminal Justice Review, 43(4), 458-476. Recuperado de https://www.scopus.com/inward/record.uri?eid=2-s2.0-85042233794\&do

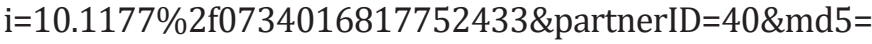

Sander, G. \& Lines, R. (2016). HIV, hepatitis C, TB, harm reduction, and persons deprived of liberty: What standards does international human rights law establish? Health and Human Rights, 18(2), 171-182. Retrieved from https://www.scopus. com/inward/record.uri?eid=2-s2.0-85003955291\&partnerID=40\&md5=589e e977534e3fabf095aac312a95388

Santorso, S. (2015). The perception of emprisonment between deprivation and solidarity. An analysis of the socio-economic conditions of prisoners. Deviance et Societe, 39(2), 171-188. Retrieved from https://www.scopus.com/inward/ record.uri?eid=2-s2.0-84975110502\&partnerID=40\&md5=9d4c68fadcd $478 \mathrm{~d}$ 0b85c5ef53ab3db10 
Saxena, A. (2020). The candle burns: human rights violations of under trial prisoners in India with particular emphasis on Madhya Pradesh. International Journal of Human Rights, 24(5), 615-631. Retrieved from https://www.scopus.com/ inward/record.uri?eid=2-s2.0-85075733737\&doi=10.1080\%2f13642987.20 19.1663341\&partner ID $=40$ \&

Schabbach, L. (2020). A militarizacao dos presidios brasileiros. Tempo Social, 32(1), 227-245. Retrieved from https://www.scopus.com/inward/record.uri?eid=2s2.0-85086833623\&doi=10.11606\%2f0103-2070.TS.2020.156940\&partnerID=

Schartmueller, D. (2015). Settling Down Behind Bars: The Extensive Use of Life Sentences in Alabama. The Prison Journal, 95(4), 449-471. Retrieved from https:// www.scopus.com/inward/record.uri?eid=2-s2.0-84945403992\&doi=10.1177 \%2f0032885515596521\&partnerID=40\&md5=

Simon, J. (2017). Beyond tough on crime: Towards a better politics of prosecution Prosecutors and Democracy: A Cross-National Study. Retrieved from https://www. scopus.com/inward/record.uri?eid=2-s2.0-85048667522\&doi=10.1017\%2f9 781316941461.010\&partnerID $=40 \&$ md5 $=$

Simon, J. (2018). Penal monitoring in the United States: lessons from the American experience and prospects for change. Crime, Law and Social Change, 70(1), 161173. Retrieved from https://www.scopus.com/inward/record.uri?eid=2-s2.085046483437\&doi=10.1007\%2fs10611-017-9724-0\&partnerID=40\&md5=

Skinns, D. (2016). Coalition government penal policy 2010-2015: Austerity, outsourcing and punishment. Retrieved from https://www.scopus.com/inward/ record.uri?eid=2-s2.0-85017620467\&doi=10.1057\%2f978-1-137-457349\&partnerID $=40 \& \mathrm{md} 5=$

Snyder, C., Van Wormer, K., Chadha, J. \& Jaggers, J.W. (2009). Older adult inmates: The challenge for social work. Social Work, 54(2), 117-124. Retrieved from https:// www.scopus.com/inward/record.uri?eid=2-s2.0-65549154248\&doi=10.1093\% 2fsw\%2f54.2.117\&partnerID=40\&md5=e5e357b78de26f4fe6e65909893ca6a9

Spencer, L. (2012). A state of emergency in Alabama: Prison overcrowding. SAGE Open, 2 (3), 1-5. Retrieved from https://www.scopus.com/inward/record. uri?eid=2-s2.0-84883232272\&doi=10.1177\%2f2158244012460695\&partne $\mathrm{rID}=40 \& \mathrm{md} 5=$

Stewart, W. (2018). What Does the Implementation of Peer Care Training in a U.K. Prison Reveal About Prisoner Engagement in Peer Caregiving?.Journal of Forensic Nursing, 14(1), 18-26. Retrieved from https://www.scopus.com/inward/record. uri?eid=2-s2.0-85042852272\&doi=10.1097\%2fjFN.0000000000000183\&par tnerID=40\&md5= DOI: $10.1097 / J F N .0000000000000183$

Sundt, J., Salisbury, E. \& Harmon, M.G. (2016). Is Downsizing Prisons Dangerous?: The Effect of California's Realignment Act on Public Safety Sundt, Salisbury, 
and Harmon Downsizing Prisons. Criminology and Public Policy, 15(2), 315341. Retrieved from https://www.scopus.com/inward/record.uri?eid=2-s2.084960153735\&doi=10.1111\%2f1745-9133.12199\&partnerID=40\&md5=6a9 ee3c6421431b5b87d4a9bfd971ebe

Tham, H. (2014). Lessons from criminal policy developments in Sweden, Scandinavia and Western Europe. En Gutiérrez, M. et al. (eds.), Política Criminal y Libertad (pp. 91-120). Bogotá: Universidad Externado de Colombia. Recuperado de https:// books.openedition.org/uec/1038

Timor, U. (2006). Privatization of prisons in Israel: Gains and risks Israel. Law Review, 39(1), 81-104. Retrieved from https://www.scopus.com/inward/record. uri?eid=2-s2.0-84917264516\&doi=10.1017\%2fS0021223700012929\&partn erID $=40 \& \mathrm{md} 5=$

Tulkens, F. (2014). Prisons in Europe: Recent developments in the jurisprudence of the European Court of Human Rights européenne des droits de l'homme. Deviance et Societe, 38(4), 425-448. Recuperado de https://www.scopus.com/inward/ record.uri?eid=2-s2.0-84937028600\&partnerID=40\&md5=b8350f921e7a59 9a18e94e8d99aa541e

Ungar, M. \& Magaloni, A. (2009). Latin America's prisons: A crisis of criminal policy and democratic rule. Criminality, Public Security, and the Challenge to Democracy in Latin America, 223-248. Retrieved from https://www.scopus.com/inward/ record.uri?eid=2-s2.0-84858642429\&partnerID=40\&md5=a1f0a069a807056 cce19d1c250d9ab0a

van Ginneken, E., Sutherland, A. \& Molleman, T. (2017). An ecological analysis of prison overcrowding and suicide rates in England and Wales, 2000-2014. International Journal of Law and Psychiatry, 50, 76-82. Retrieved from https://www.scopus. com/inward/record.uri?eid=2-s2.0-84967166366\&doi=10.1016\%2fj.ijlp.2016 .05.005\&partnerID=40\&md5=5059a0eb38074f163b6d2105d23315d0

Zanis, D., Mulvaney, F., Coviello, D., Alterman, A.I., Savitz, B. \& Thompson, W. (2003). The effectiveness of early parole to substance abuse treatment facilities on 24-month criminal recidivism. Journal of Drug Issues, 33(1), 223-235. Retrieved from https://www.scopus.com/inward/record.uri?eid=2-s2.0-0037412804\&do

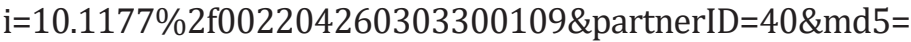

Zevallos, J. (2016). Overcrowding in the Peruvian prison system International. Review of the Red Cross, 98(903), 851-858. Recuperado de https://www.scopus.com/ inward/record.uri?eid=2-s2.0-85044455342\&doi=10.1017\%2fS1816383117 000649\&partnerID $=40 \&$ md5 $=$ 